University of Nebraska - Lincoln

DigitalCommons@University of Nebraska - Lincoln

Faculty Publications in Computer \& Electronics Electrical \& Computer Engineering, Department Engineering (to 2015)

2012

Performance Analysis for Direction of Arrival Estimating Algorithms

\author{
Pradhumna Shrestha \\ University of Nebraska Lincoln \\ Michael Hempel \\ University of Nebraska Lincoln, mhempel2@unl.edu \\ Puttipong Mahasukhon \\ University of Nebraska-Lincoln, pmahasukhon@huskers.unl.edu \\ Tao Ma \\ University of Nebraska Lincoln, tma@unlnotes.unl.edu \\ Hamid Sharif \\ University of Nebraska-Lincoln, hsharif@unl.edu
}

Follow this and additional works at: https://digitalcommons.unl.edu/computerelectronicfacpub

Part of the Computer Engineering Commons

Shrestha, Pradhumna; Hempel, Michael; Mahasukhon, Puttipong; Ma, Tao; and Sharif, Hamid, "Performance Analysis for Direction of Arrival Estimating Algorithms" (2012). Faculty Publications in Computer \& Electronics Engineering (to 2015). 85.

https://digitalcommons.unl.edu/computerelectronicfacpub/85

This Article is brought to you for free and open access by the Electrical \& Computer Engineering, Department of at DigitalCommons@University of Nebraska - Lincoln. It has been accepted for inclusion in Faculty Publications in Computer \& Electronics Engineering (to 2015) by an authorized administrator of DigitalCommons@University of Nebraska - Lincoln. 


\title{
Performance Analysis for Direction of Arrival Estimating Algorithms
}

\author{
Pradhumna Lal Shrestha, Michael Hempel, Puttipong Mahasukhon, Tao Ma and Hamid Sharif \\ Computer and Electronics Engineering Department \\ University of Nebraska - Lincoln, Omaha, NE 68182 \\ \{plshrestha, mhempel, pmahasukhon, tma, hsharif \}@unlnotes.unl.edu
}

\begin{abstract}
Smart antennas have emerged as one of the most promising directions in supporting maximum communication link throughput. In this paper, we have investigated the impact of smart antennas on a complex mobile network such as a railroad wireless communications system. The objective is to analyze the selection of a Direction-Of-Arrival (DOA) estimation algorithm which provides the maximum efficiency when deployed in our railroad testbeds for wireless vehicular communication. Our findings are discussed to provide an indepth understanding of how different algorithms should be selected to support efficient network operations.
\end{abstract}

Keywords- Direction of Arrival, Railroads, MUSIC, ESPRIT, Wireless, Mobile, Vehicular Networks

\section{INTRODUCTION}

In any communication system, it is desirable to deliver maximum throughput. In achieving this objective, many hardware, physical layer and cross layer design techniques have been studied in the literature. Among these approaches, smart antennas are one of the most promising directions.

Smart antennas have two primary aspects: position estimation and beamforming. An array of antenna elements is employed to receive multiple versions of the same signal from a distant source, at slightly different locations. The signals are then processed to indicate the position of the source. In a radial coordinate system, the azimuthal and elevation angles represent that position. Then, the array steers the principal lobe of the beam pattern towards the estimated Direction-of-Arrival (DOA). This way, the maximum power emitted by the antenna is directed towards the desired source, resulting in a very high effective antenna gain in that direction.

In [1], the authors show that the throughput can be improved by using smart antennas in a network. However, when designing a communication infrastructure for complicated networks and testbeds, the mathematical derivation and analysis does not provide all the details. Therefore, use of testbed simulation tools to predict smart antenna performance is needed.

Our research team at the Advanced Telecommunications Engineering Laboratory at the University of Nebraska-Lincoln has been studying the performance of high-speed networks for mobile railroad $[2,3]$ and other vehicular environments. In our previous works $[2,3]$, we have extensively analyzed different wireless communication systems for railroad trackside and yard deployments. Our work includes theoretical analysis as well as simulation studies using NS-2 and MATLAB. Our theoretical work was supported by measured data from our testbeds [4]. The work presented here is to analyze the impact of smart antennas on a complex mobile railroad environment

The initial step and one of the key aspects in integrating smart antenna systems into our previous works is the selection of an appropriate Direction-of-Arrival algorithm.

Many research works have investigated different designs of a DOA estimation algorithm. The choice of an algorithm depends on the specifications and requirements of the project itself. In our study, we can be flexible for antenna parameters like number of antenna elements and number of source samples used for estimation.

In $[5,6]$, the authors have studied integrating smart antennas into NS-2 but they cannot be directly applied to railroads communication networks. The reason is that estimation of radiation pattern and gain of the antenna system in complex railroad networks cannot be simply evaluated analytically. The outcomes are severely affected by the interaction between the antenna's electromagnetic radiation and metallic elements in the train or wayside equipment construction. The gain produced by the antenna system depends not only on the property of the antenna but also on its location within the environment. Thus the benefit of using directional smart antennas in terms of gain is better evaluated by computer simulation or field testing.

In spite of the plethora of algorithms proposed to estimate the Direction-of-Arrival, only few works have been published that compare these algorithms, especially the two-dimensional estimation algorithms. Such a comparison, however, is essential for the proper selection of an algorithm for future devices and technologies. In this paper we have presented the evaluation of three sample Direction-of-Arrival estimation algorithms [7, 8]. Section II introduces the algorithms with discussions of their advantages and weaknesses. Section III explains the procedures we implemented. Section IV shows the results of our simulations. Finally, Section V concludes our work.

\section{SAMPLE ALGORITHMS}

\section{A. MUSIC}

In [9], Schmidt revolutionized the idea of direction of arrival estimation with his original MUSIC algorithm. The major contribution of the method was the ability to handle arbitrary configurations of antenna arrays, thus relieving the designer 


\begin{tabular}{|c|c|c|c|c|}
\hline SNR $(\mathrm{dB})$ & Source Samples & Angles & Separation & Power Ratio \\
\hline 0 & 500 & $\left(10^{\circ}, 20^{\circ}\right),\left(20^{\circ}, 30^{\circ}\right)$ & $10^{\circ}$ & 1 \\
\hline 2.5 & 750 & $\left(10^{\circ}, 20^{\circ}\right),\left(25^{\circ}, 35^{\circ}\right)$ & $15^{\circ}$ & 2 \\
\hline 4 & 800 & $\left(10^{\circ}, 20^{\circ}\right),\left(28^{\circ}, 38^{\circ}\right)$ & $18^{\circ}$ & 4 \\
\hline 5 & 900 & $\left(10^{\circ}, 20^{\circ}\right),\left(30^{\circ}, 40^{\circ}\right)$ & $20^{\circ}$ & 5 \\
\hline 7.5 & 1000 & $\left(10^{\circ}, 20^{\circ}\right),\left(35^{\circ}, 45^{\circ}\right)$ & $25^{\circ}$ & 7 \\
\hline 8 & 1250 & $\left(10^{\circ}, 20^{\circ}\right),\left(37^{\circ}, 47^{\circ}\right)$ & $27^{\circ}$ & 8 \\
\hline 10 & 1300 & $\left(10^{\circ}, 20^{\circ}\right),\left(40^{\circ}, 50^{\circ}\right)$ & $30^{\circ}$ & 9 \\
\hline 12.5 & 1400 & $\left(10^{\circ}, 20^{\circ}\right),\left(45^{\circ}, 55^{\circ}\right)$ & $35^{\circ}$ & 10 \\
\hline 15 & 1500 & $\left(10^{\circ}, 20^{\circ}\right),\left(50^{\circ}, 60^{\circ}\right)$ & $40^{\circ}$ & \\
\hline
\end{tabular}

Table 1: Parameters used for simulation

from the constraint of building an array with a fixed specific sensitivity pattern. After its initial publication the algorithm has undergone many modifications. Though the original paper only deals with one-directional DOA estimation, we have modified it by includind the second dimension to produce both angles.

The MUSIC spectrum is defined by,

$$
F(\theta, \phi)=\frac{A(\theta, \phi)^{H} A(\theta, \phi)}{A(\theta, \phi)^{H} V_{N} V_{N}^{H} A(\theta, \phi)}
$$

where, $\theta$ and $\Phi$ are the elevation and azimuthal angles of the source position in a radial co-ordinate system, $\mathrm{A}(\theta, \Phi)$ is the array steering matrix defined by the antenna array's relative position with respect to the signal source, $\mathrm{V}_{\mathrm{N}}$ represents the eigenvectors corresponding to the noise space of the received signal and [.] ${ }^{\mathrm{H}}$ represents the Hermitian transpose of a matrix.

The spectrum is clearly maximized at places where noise space and steering matrix are orthogonal to each other. Therefore, the values of $(\theta, \Phi)$ at the corresponding spectrum peaks provide the direction of arrival.

Since MUSIC computes the spectrum to separate noise and signal spaces at every possible values of $(\theta, \Phi)$, it involves an extensive search procedure, making it computationally very expensive. But its high accuracy and flexibility makes it an interesting prospect for our evaluation.

\section{B. ESPRIT and ESPRIT-like methods}

Roy and Kailath in [10] suggested another algorithm for DOA estimation, which uses a complex geometry of the antenna array by imposing requirements on the array structure, but in return was computationally much more efficient than MUSIC. Since the time of its publication, different versions of ESPRIT and ESPRIT-like algorithms have been published.

In [8], Wu et al. suggested an algorithm for two dimensional DOA estimation based on a propagator method. Though the algorithm did not require any eigenvalue decomposition or singular value decomposition (SVD) and was computationally efficient, there was the need to pair the azimuth and elevation angles and any failure to pair them appropriately would fail the algorithm. Liu and Mendel have described the algorithm used for pairing the two direction of arrival in [12].

To overcome this, Liang, in [7], introduced a cumulantbased approach for two-dimensional DOA estimation that generates the angles in pairs, but has higher computational complexity and uses a special volume array. It is based on a parallel factor (PARAFAC) [13] model of cumulant matrices in the cumulant domain. The fourth order cumulant is defined as cumulant $(\mathcal{A}, \mathcal{B}, \mathcal{C}, \mathcal{D})$

$=E(\mathcal{A} * \mathcal{B} * \mathcal{C} * \mathcal{D})-E(\mathcal{A} * \mathcal{B}) E(\mathcal{C} * \mathcal{D})$

$-E(\mathcal{A} * \mathcal{C}) E(\mathcal{B} * \mathcal{D})-E(\mathcal{A} * \mathcal{D}) E(\mathcal{B} * \mathcal{C})$

Where, $A, B, C$ and $D$ are arbitrary matrices, $\mathrm{E}[$.] is the expectation operator and * represents component-wise product of the matrices, The algorithm in [7] also has several benefits over any published algorithm.

\section{Algorithm ANALYSIS AND EXPERIMENT}

The three algorithms, mentioned in Section II, were simulated in MATLAB using arbitrarily chosen elevation and azimuth angles in an AWGN channel. First, the source location was fixed at specific angles and a random signal generator was simulated at the source location. This setup was chosen to ensure minimal correlation between the data points. The receiving antenna was arranged as a square array for evaluating MUSIC. The remaining two algorithms were arranged as specified in their respective publication. We simulated the algorithms in [7, 8] 500 times for every set of angles for each condition. The errors of estimation for each angle were calculated for every simulation and the results were used to calculate the mean and variance for each test set. Due to the computational complexity of MUSIC, it was performed only 5 times but we believe that the results will not be deeply affected if it was simulated more often than the other algorithms due to the precision and stability of MUSIC's results.

For simulating various channel conditions, we chose SNR values as shown in column I of table 1 . Two sources were fixed with direction of arrival $\left(10^{\circ}, 20^{\circ}\right)$ and $\left(30^{\circ}, 40^{\circ}\right)$. We set the number of array elements for each system at 9 and number of source samples at 1000 .

For varying the number of source samples, we opted for the sample counts shown in column II of table 1 and a fixed SNR set at $5 \mathrm{~dB}$. The rest of the conditions are kept the same as above. 


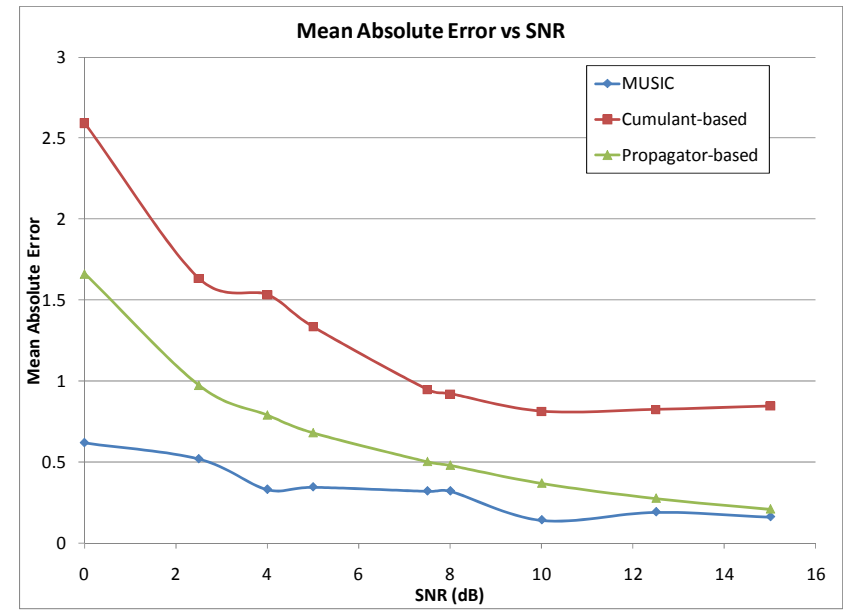

Figure 1: Effect of SNR on mean of estimation error of angle of arrival

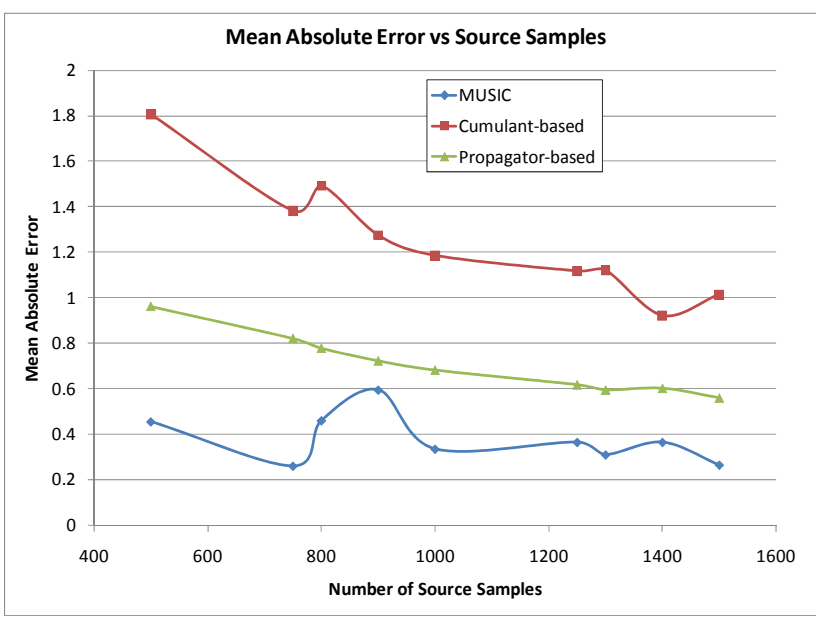

Figure 3: Effect of number of source samples on mean of estimation error of angle of arrival

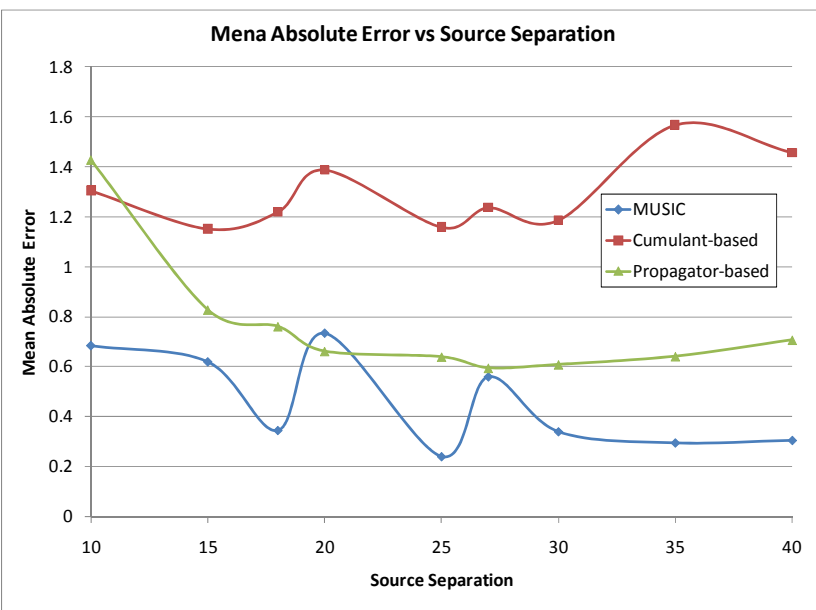

Figure 5: Effect of source separation on mean of estimation error of angle of arrival

For simulating angular separation between sources, the SNR was fixed at $5 \mathrm{~dB}$ and the number of source samples at 1000 . Two sources were chosen with direction of arrival as shown in

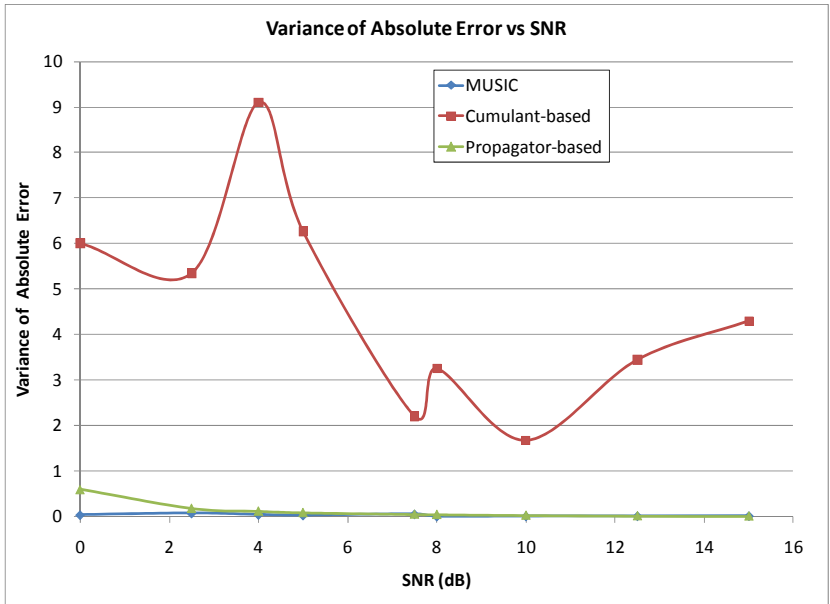

Figure 2: Effect of SNR on variance of estimation error of angle of arrival

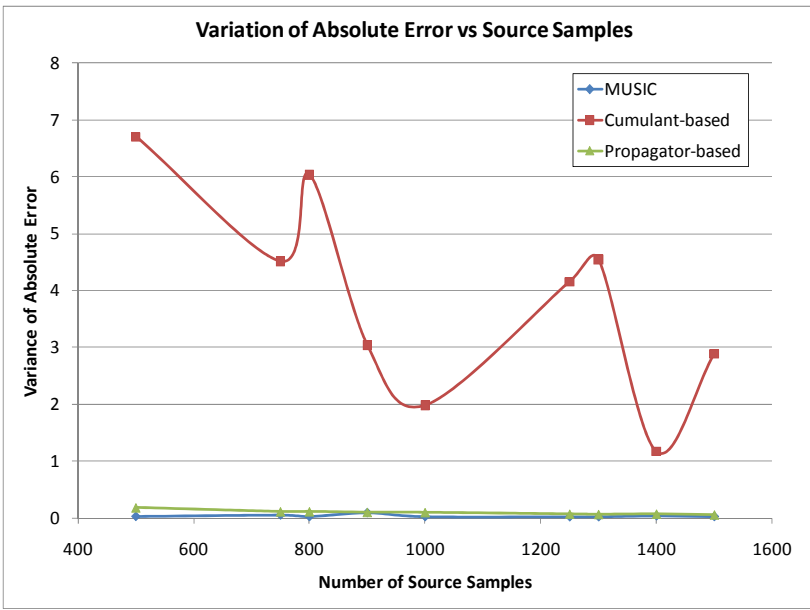

Figure 4: Effect of number of source samples on variance of estimation error of angle of arrival

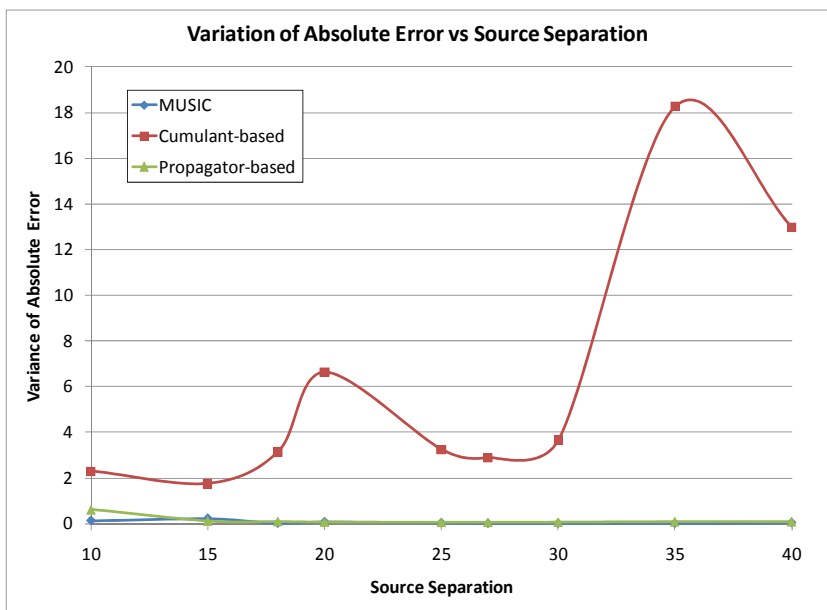

Figure 6: Effect of source separation on variance of estimation error of angle of arrival

column III of table 1, with corresponding separation in column IV. 


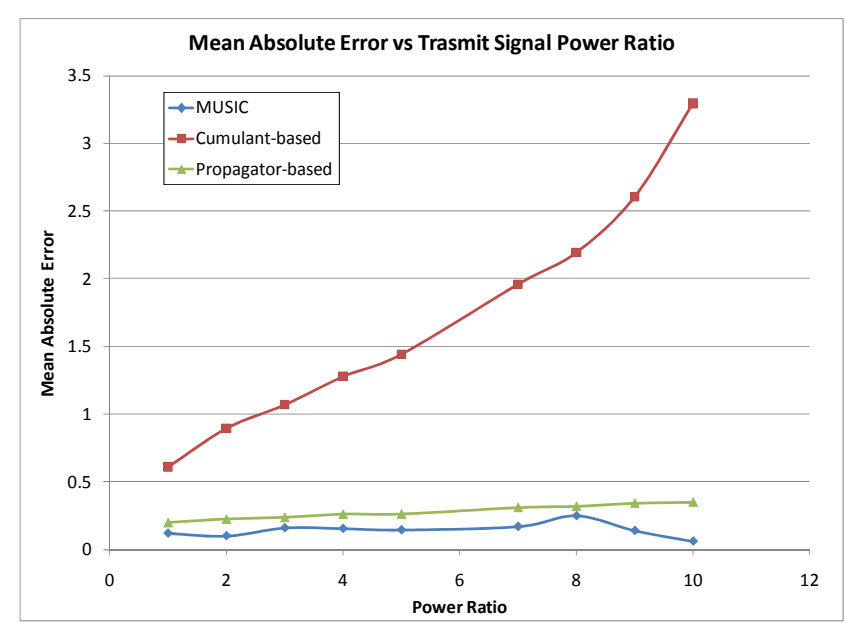

Figure 7: Effect of transmit signal power ratio on mean of estimation error of angle of arrival

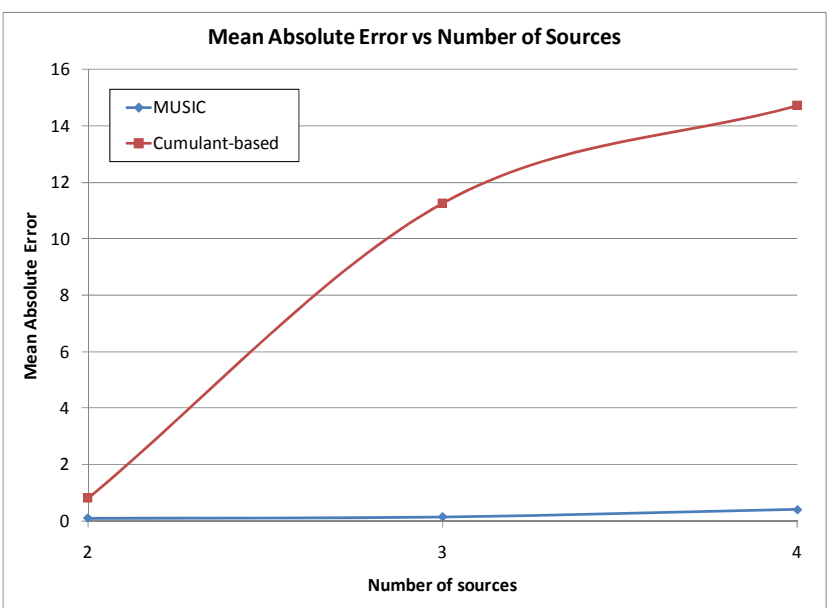

Figure 9: Effect of number of sources on estimation error of angle of arrival

To simulate the near-far effect, or cases where the two sources are radiating at different power levels, we chose a fixed SNR of $15 \mathrm{~dB}$, the number of samples as 1000 , and direction of arrival at $\left(10^{\circ}, 20^{\circ}\right)$ and $\left(30^{\circ}, 40^{\circ}\right)$. We then varied the power ratio of the source at $\left(30^{\circ}, 40^{\circ}\right)$ relative to that at $\left(10^{\circ}, 20^{\circ}\right)$. We selected the ratio as shown in column $\mathrm{V}$ of table 1.

Finally, we also simulated the effect of increasing the number of sources. The algorithm in [8] was not used for this purpose to retain the same number of antenna array elements as 9. For simulations requiring three sources we chose the directions to be at $\left(10^{\circ}, 20^{\circ}\right),\left(30^{\circ}, 40^{\circ}\right)$ and $\left(50^{\circ}, 60^{\circ}\right)$. For scenarios with four sources we selected to add a direction of $\left(60^{\circ}, 70^{\circ}\right)$. The SNR was fixed at $15 \mathrm{~dB}$ and the number of source samples set to 1000 .

\section{RESULTS}

The effect of estimation of direction of arrival due to variation on number of source samples, signal to noise ratio, angular separation between the sources, transmitter power variation and number of sources to be estimated are shown in Figures 1-10. Figures 1, 3, 5, 7 and 9 show the mean of

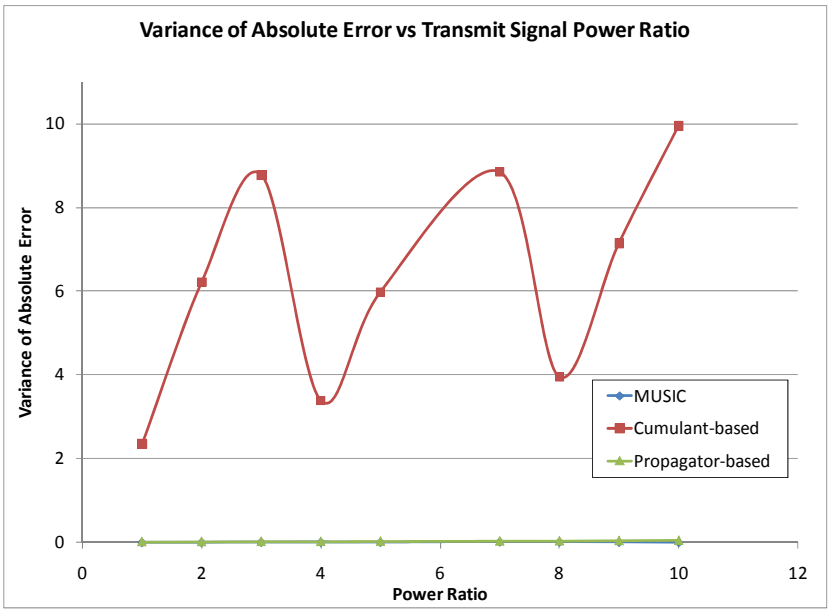

Figure 8: Effect of transmit signal power ratio on variance of estimation error of angle of arrival

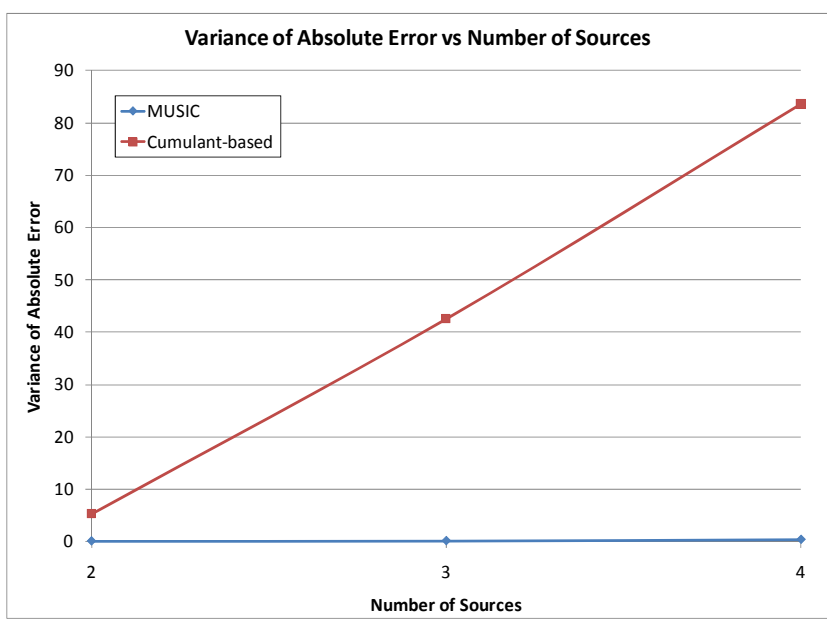

Figure 10: Effect of number of sources on variance of estimation error of angle of arrival

absolute error and Figures 2, 4, 6, 8 and 10 show the variance of absolute error.

Some of the figures are non-monotonic, which is contrary to theoretical expectations. This can be attributed to two causesthe algorithm itself and the simulation scenario. For the simulated channel conditions, the ESPIRIT based algorithms are known to sometimes fail. These failures negatively impact both the mean and the variance of our results. We decided to retain these data results instead of removing them, however, because we feel this fluctuation truly reflects the behavior of the algorithm for the simulated conditions. Furthermore, a random signal generator is not guaranteed to match the desired statistics of mean and variance when a finite, albeit large, number of data samples is used. Therefore, parameters such as SNR fluctuate for each simulation point and can sometimes significantly divert from the desired value, albeit with low probability. This fluctuation in the simulation scenario also contributed to the non-monotonocity of the curves.

As expected, with better channel conditions and better information about source location, the accuracy in estimation improves. On first glance, even though the results of ESPRIT- 
based algorithms look as good as those of MUSIC, they have been averaged over multiple simulations and have large variances. The relative stability and accuracy of MUSIC makes it attractive for complex networks such as the one in our study. To put this into a quantitative perspective, if we sample the source moving linearly at 20 meters per second (about $45 \mathrm{mph}$ ) at the rate of 1 Msps, for a block of 1000 source samples we need to sample for $1 \mathrm{~ms}$ during which the source will move a linear distance of $20 \mathrm{~mm}$ which is insignificant and will not affect the accuracy of estimation. However with ESPRIT we would need hundreds of sample block sets (500 used for simulation), which effectively means that the source would move by a linear distance of $10 \mathrm{~m}$ by the times we finish sampling. This value of $10 \mathrm{~m}$ can introduce severe errors in accuracy, particularly if the communicating end-points are very near to each other. Only if they are sufficiently far enough apart $(>1 \mathrm{~km})$, the change in angles may be neglected. However, the computational complexity of MUSIC is much higher than that for ESPRIT-based algorithms, requiring more complex hardware and a larger computational energy budget. In situations of computational or energy constraints, MUSIC is therefore not the best choice and ESPRIT-based algorithms should be favored for their much better complexity and energy cost, especially if the compromise in accuracy, as explained in the earlier example, is deemed insignificant for the targeted application.

As we can see, the performance advantages of one algorithm over another vary with the conditions, both of the environment as well as the system. Thus, there is no clear favorite among the algorithms and careful consideration needs to be given to the conditions and system parameters specific to the planned deployment.

\section{CONCLUSION AND FUTURE WORK}

This paper investigated the efficiency of widely available Direction-of-Arrival algorithms for application in complex networks such as our railroad testbeds. In Section IV, we showed that MUSIC is the more effective option to use for high velocity sources and poor channel conditions as it does not require multiple estimates for the same pair of angles due to its relative accuracy and stability. However, if source velocity is not very high and multiple estimations of the direction of arrival are possible, other discussed methods should be used to save computational complexity.

For our testbeds, communication between the relatively stationary networks ESPRIT-like algorithms will be effective while for railcars to base-station communication, using MUSIC would be more appropriate.

Our future work will include estimating array gain coefficients using suitable beamforming algorithms and simulate the testbed in CST [11] to achieve exact gain produced by using smart antennas under our test conditions.

\section{ACKNOWLEDGEMENT}

This work was supported in part by NSF award CNS1065069.

\section{REFERENCES}

[1]. C.A. Balanis, A.S. Spania, T.M.Duman and Capone, J.M., "Impact of smart antenna designs on network throughput and BER", Antennas and Propagation Society International Symposium, 2004.

[2]. Michael Hempel, Hamid Sharif, Wei Wang, Ting Zhou, Puttipong Mahasukhon, "A Study of Critical Baseline Performance Characteristics for IEEE 802.16e-2005”, IWCMC 2008

[3]. P. Mahasukhon, H. Sharif, M. Hempel, T. Zhou, W.Wang, H.-H. Chen, "IEEE $802.11 \mathrm{~b}$ based ad hoc networking and its performance in mobile channels", Communications, IET 2009

[4]. Puttipong Mahasukhon, Hamid Sharif, Michael Hempel, Ting Zhou, and Tao Ma, "Distance and Throughput Measurements in Mobile WiMAX Test Bed", MILCOM 2010

[5]. F. Babich, M. Comisso, A. Dorni, F. Barisi, F. and A. Mania, "The Simulation of Smart Antennas in Network Simulator-2 Using MATLAB", Computer Aided Modeling and Design of Communication Links and Networks, 2009

[6]. F. Babich, M. Comisso, A. Dorni and F. Barisi, "Open source simulation of smart antenna systems in network simulator-2 using Octave", Wireless Pervasive Computing (ISWPC), 2010

[7]. Junli Liang, 'Joint Azimuth and Elevation Direction Finding Using Cumulant”, IEEE SENSORS JOURNAL, VOL. 9, NO. 4, APRIL 2009

[8]. Yuntao Wu, Guisheng Liao and H.C. So, “ A Fast Algorithm for 2-D Direction of Arrival Estimation", SIGNAL PROCESSING 83 (2003) $1827-1831$

[9]. Ralph O. Schmidt, "Multiple Emitter Location and Signal Parameter Estimation", IEEE TRANSACTIONS ON ANTENNAS AND PROPAGATION, VOL. AP-34, NO. 3, MARCH 1986

[10]. Richard Roy and Thomas Kailath, "ESPRIT-Estimation of Signal Parameters Via Rotational Invariance Techniques" TRANSACTIONS ON ACOUSTICS. SPEECH. AND SIGNAL PROCESSING. VOL 37. NO 7. JULY 1989

[11]. CST Computer Simulation Technology AG, Germany, homepage: www.cst.com

[12]. Tsung-Hsien Liu and Jerry M. Mendel, "Azimuth and Elevation Direction Finding Using Arbitrary Array Geometries", IEEE TRANSACTIONS ON SIGNAL PROCESSING, 1998

[13]. N. D. Sidiropoulos, "COMFAC: Matlab Code for LS Fitting of the Complex PARAFAC Model in 3-D”, http://www.telecom.tuc.gr/ nikos 